\title{
Global Stability of a Class of Discontinuous Dual Billiards
}

\author{
Franco Vivaldi ${ }^{1}$ and Anna V. Shaidenko ${ }^{2}$ \\ ${ }^{1}$ School of Mathematical Sciences, Queen Mary College, Mile End Road, London E1 4NS, \\ England \\ 2 Department of Mathematics, SU-630090 Novosibirsk, USSR
}

\begin{abstract}
An infinite-parameter family of discontinuous area-preserving maps is studied, using geometrical methods. Necessary and sufficient conditions are determined for the existence of some bounding invariant sets, which guarantee global stability. It is shown that under some additional constraints, all orbits become periodic, most of them Lyapounov stable, and with a maximal period in any bounded domain of phase space. This yields a class of maps acting on a discrete phase space.
\end{abstract}

\section{Introduction}

In this work we shall be concerned with the stability problem of an infiniteparameter family of piecewise linear discontinuous area-preserving maps. At the same time, we intend to exploit the peculiar properties of this class of systems to reproduce in a simplified form some features of smooth nonintegrable transformations.

A generic smooth canonical map of the plane is known to possess both regular and irregular orbits. Regular orbits correspond to irrational rotations on invariant circles, due to the KAM theorem [1], whereas the irregular ones, which develop in the place of rational rotations, are still not fully understood. It is known, however, that about transverse homoclinic intersections of separatrices, hyperbolic invariant sets exist over which the map reduces to a shift [1]. The truly random evolution which characterizes this class of solutions has placed them beyond the scope of modern analytical methods.

The main difficulty to be overcome is rooted in the very definition of dynamical instability, which is essentially equivalent to asymptotic $(|t| \rightarrow \infty)$ discontinuous dependence of the solutions upon the initial conditions [2]. This property, shared not only by all unstable periodic orbits, but rather by the entire set of intersecting separatrices, is the source of that infinite variety of topologically distinct solutions which is a synonym for random dynamics. An additional complication stems from the fact that in smooth nonintegrable systems regular and irregular motions always form a nested pattern repeating itself on every scale. 
In an integrable system most solutions are rotations, yet its topological characterization derives from the zero measure set of separatrices. Thus, from a topological viewpoint, an integrable system and a small perturbation of it are never close to each other, due to the dramatic evolution of separatrices under the influence of a perturbation. It is therefore unclear to what extent, if any, irregular motions can be reproduced by means of sufficiently complicated regular ones. The asnwer to this question is known to be negative when an integrable system is obtained from a nonintegrable one by truncating its Birkhoff normal form (or similar asymptotic series of canonical transformations), which is the only systematic approach to this problem available to date [3]. These methods are illfounded since they attempt to force upon the perturbed system the simple features of the unperturbed one.

One way of circumventing these pathological obstacles is to consider nonsmooth systems, to which the KAM theorem does not apply. For instance, it has been recently shown that non-differentiable maps display some features of nonintegrable systems in a more manageable form, and at the same time they can still exhibit bounding invariant curves $[4,5]$. Furthermore, a class of nonintegrable discontinuous plane billiards are known to possess (non-toroidal) invariant integral surfaces, which yield a remarkable simplification in the geometry of the phase space [6-8].

Here we shall consider discontinuous canonical maps of the plane, constructed geometrically by means of convex polygons (see Sect. 2). The purpose of our investigation is three-fold:

1. Establish that, in spite of the discontinuity, isolating invariant sets can exist, which afford the formulation of a criterion for global stability in closed form.

2. Show that under certain assumptions, these systems can be made fully periodic, and with a finite number of topologically distinct orbits in any bounded region of the phase space. Due to this simplification, the entire structure of the phase space can then be described via finite computation.

3. Demonstrate that discontinuous maps are very efficient tools for exploring the roots of irregular motion, for its true source, namely the discontinuity, is here explicitly exposed.

This paper is organized as follows. In Sect. 2 we define the family of maps to be studied, and in Sect. 3 we establish some of their properties. A criterion for global stability is formulated in Sect. 4, while in Sect. 5 we obtain conditions for the full periodicity of the system. Finally, in Sect. 6 we present concluding remarks.

\section{Formulation of the Problem}

We consider a map $\phi$ of the exterior $E$ of an oriented closed plane convex curve $\Gamma$ onto itself(Fig. 1). The mapping $\phi$ is defined as follows $[1,9]$ : from any point $p_{0} \in E$ we draw the tangent $L$ to $\Gamma$, denoting by $q$ the point of contact. The line $L$ is selected among the two possible tangents according to the orientation of $\Gamma$. We denote by $\phi\left(p_{0}\right)$ the point obtained by reflecting $p_{0}$ with respect to $q$. [If the segment $L \cap \Gamma$ is non-degenerate, we denote by $q$ its midpoint, because only in this case does $\phi(E)=E$.] Similarly, we define the inverse image $p_{0}=\phi^{-1}\left(p_{1}\right)$, where $p_{1}=\phi\left(p_{0}\right)$. 


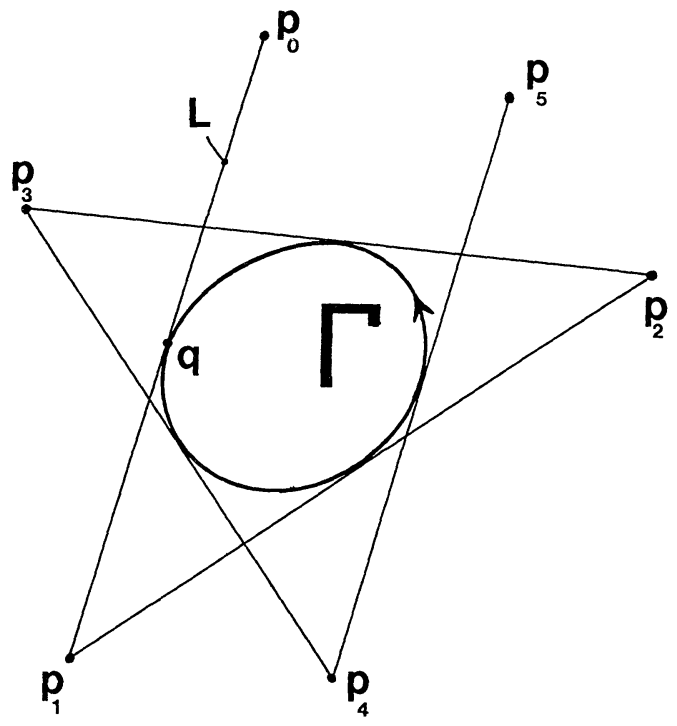

Fig. 1. Construction of the map $\phi$

The collection $\left\{p_{k}=\phi^{k}\left(p_{0}\right)\right\}, k \in \mathbb{Z}$, is called an orbit through $p_{0}$. The map $\phi$ constructed in this way is uniquely determined by $\Gamma$. It can be shown that these transformations are area-preserving, and that affine equivalent curves generate affine equivalent orbits [9]. Thus we have an infinite-parameter family of canonical maps which we call dual billiards.

Dual billiards have been proposed as crude models for orbital motions $[1,10]$, and the main problem is to establish whether or not all orbits are bounded. This problem is easily solved in the only known integrable case, namely the circle, where all orbits lie on circles. In nonintegrable cases, one seeks to find bounding invariant curves, located at sufficient distance from $\Gamma$. The existence of these curves can actually be demonstrated via the KAM theorem, provided that $\Gamma$ is six times continuously differentiable [1]. If such boundaries do not exist, then some orbits could disperse to infinity following spirals of increasing radius.

In what follows we shall restrict ourselves to systems for which $\Gamma$ is a polygon; these systems yield discontinuous mappings, as evident.

\section{Basic Properties}

Let $\Gamma$ be a generic convex $N$-gon, and $\Gamma_{k}, k=1,2, \ldots, N$, its sides. We begin by constructing the set $W \subset E$ of straight lines over which either $\phi$ or $\phi^{-1}$ are discontinuous (Fig. 2). If we denote by $W_{k}$ the line tangent to the $k^{\text {th }}$ side, then $W=\bigcup_{k=1}^{N} W_{k}$, where for convenience $\Gamma$ itself has been included in $W$. Let $S$ be an open domain in $E$. If $S$ does not intersect $W$, both $\left.\phi\right|_{S}$ and $\left.\phi^{-1}\right|_{S}$ are merely rigid transformations of $S$, a reflection with respect to one vertex of $\Gamma$. On the other hand, if $S$ does intersect $W$, not all its points are reflected with respect to the same 


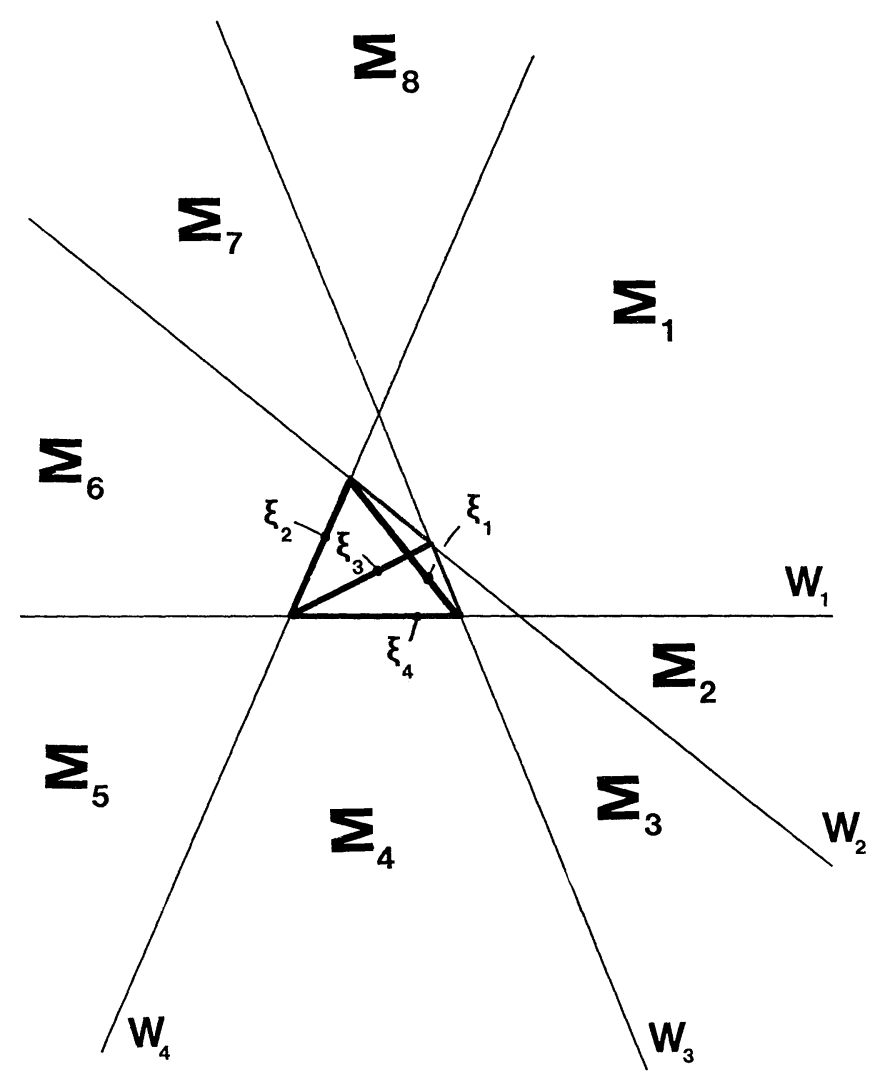

Fig. 2. The set of tangent lines $W_{k}$ for a quadrilateral, with the corresponding sectors $\Sigma_{k}$

vertex, so that $S$ is cut into two or more pieces. This mechanism constitutes the only means of deforming area elements of the plane, and hence of distinguishing the evolution of initially close trajectories. For this reason it is useful to consider the set $T$ of all those points that will eventually land on $W$, namely

$$
T=\bigcup_{k=-\infty}^{\infty} \phi^{-k}(W) .
$$

$T$ is an invariant set (by definition), consisting of lines, rays and segments which design an intricate web on the plane. Some examples of the set $T$ are depicted in Figs. $3-5,7$. We remark that the points on $T$ are the only ones that will eventually experience the discontinuity of the map, and therefore they correspond to the set of separatrices in smooth systems.

We first note that the set $T$ is connected. Indeed $W=\phi^{0}(W)$ is connected and $\phi^{n}(W)$ is obtained from $\phi^{n-1}(W)$ by adding a finite set of rays and segments with ends belonging to the set $\phi^{n-1}(W)$. Now let $S$ be the complement of $T$ in $E$. Then $S$ is disconnected. We shall regard $S$ as the collection of its connected components: $S=\bigcup_{i} S_{i}$. Each $S_{i}$ is itself a convex polygon, and it can at most have $2 N$ sides. The Lebesgue measure $\mu\left(S_{i}\right)$ of $S_{i}$ is bounded from above [see (4.9)], but in general it can 
also vanish, that is, some $S_{i}$ 's may be segments or points. All points of a given $S_{i}$ generate a bundle of orbits which always maintain their relative position, and therefore they should be regarded as a whole.

Of particular interest are those $S_{i}$ 's having positive measure, which enjoy some remarkable properties.

Lemma. If $\mu\left(S_{i}\right)>0$, and an orbit of some point in $S_{i}$ is bounded, then orbits of all points in $S_{i}$ are periodic and Lyapounov stable.

Since all points of $S_{i}$ move in the same way, the orbit of the whole $S_{i}$ is bounded. Let $D$ be a bounded domain containing all images of $S_{i}$. Then for some $k, k^{\prime}>0$ two images $\phi^{k}\left(S_{i}\right)$ and $\phi^{k^{\prime}}\left(S_{i}\right)$ must intersect, since $\mu\left(S_{i}\right)>0$. Now we note that $\phi^{k}\left(S_{i}\right)$ and $\phi^{k^{\prime}}\left(S_{i}\right)$ must coincide, although not necessarily pointwise. Indeed the boundary of $\phi^{k}\left(S_{i}\right)$ cannot intersect $\phi^{k^{\prime}}\left(S_{i}\right)$ (and vice versa), since it belongs to $T$. Both assertions of the lemma now follow from the fact that on $S_{i}$ the composed map $\phi^{2\left(k-k^{\prime}\right)}$ is just the identity. In particular, all orbits in $S_{i}$ are periodic with period equal to some divisor of $2\left(k-k^{\prime}\right)$. Q.E.D.

From the above considerations we can also say that if $S_{i}$ is periodic with period $k$ and $k$ is even, then all its points have the same period $k$, whereas if $k$ is odd then all points in $S_{i}$ have period $2 k$, except the center of symmetry of $S_{i}$, which has period $k$.

We now illustrate some easily solvable cases [10]. The simplest nontrivial polygon is just a single line segment. In this case $T=W$ is a straight line, and every orbit in $S$ escapes to infinity along a pair of straight lines parallel to $T$, while orbits in $T$ are periodic with period 2. The displacement of all points (in $S$ ) under $\phi^{2}$ is the same, and equal to twice the length of $\Gamma$. Thus the line segment is the generator of rectilinear and uniform motions.

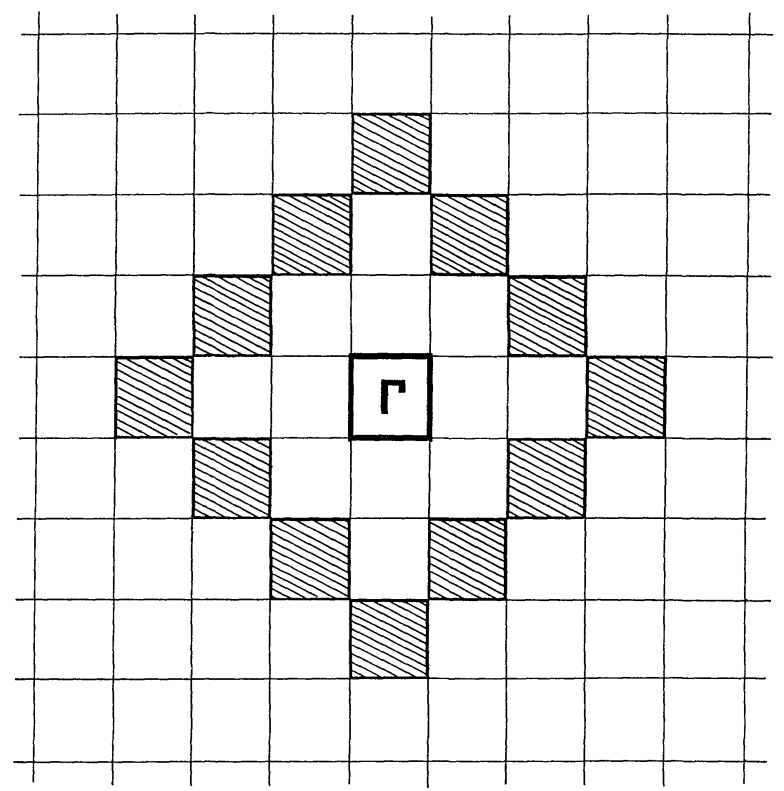

Fig. 3. The set $T$ for the square, with an invariant annulus 


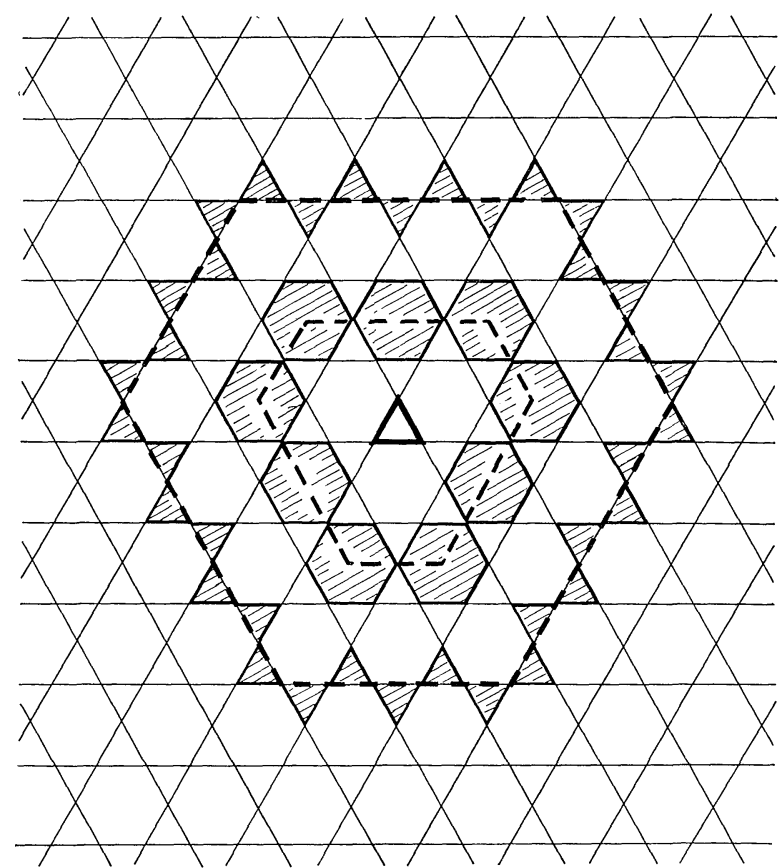

Fig. 4. The set $T$ for the triangle, with invariant annuli of the first (inner) and second (outer) kind. The corresponding integral polygons $\delta_{i}$ are represented as dashed lines

For the square, the lines $W_{k}$ are pairwise parallel, so that $T$ is an orthogonal grid dividing the plane into squares of the same size as $\Gamma$ (Fig. 3). All orbits are periodic, with periods $4 n, n=1,2, \ldots$, and they close after one complete revolution around $\Gamma$. All points having the same period form concentric invariant annuli constituted by $4 N$ squares joined at vertices. These annuli model nonlinear resonances of order $4 N$. To make this similarity more precise, we can regard the square as the limit of a sequence of smooth curves, say $x^{2 m}+y^{2 m}=1$, where $m$ is a positive integer. When $m$ is sufficiently large, the points in the center of the squares are elliptic periodic points: as $m \rightarrow \infty$ their rotation number [11] goes to zero. Similarly, the points of contact of adjacent squares in an annulus are the associated hyperbolic periodic points, and the element of $T$ emanating from them are the corresponding limit eigendirections.

In the case of a triangle, the plane is divided into triangular and hexagonal regions, whose points have period $12 n$ and $6(2 n-1), n=1,2, \ldots$, respectively (Fig. 4). Also in this case we can divide the plane into invariant annuli, of two different kinds. Finally, the set $T$ for the regular hexagon is identical to that of the triangle.

\section{Stability}

In this section we shall provide necessary and sufficient conditions for the existence of an infinite array of invariant annuli, for polygons with an arbitrarily large 


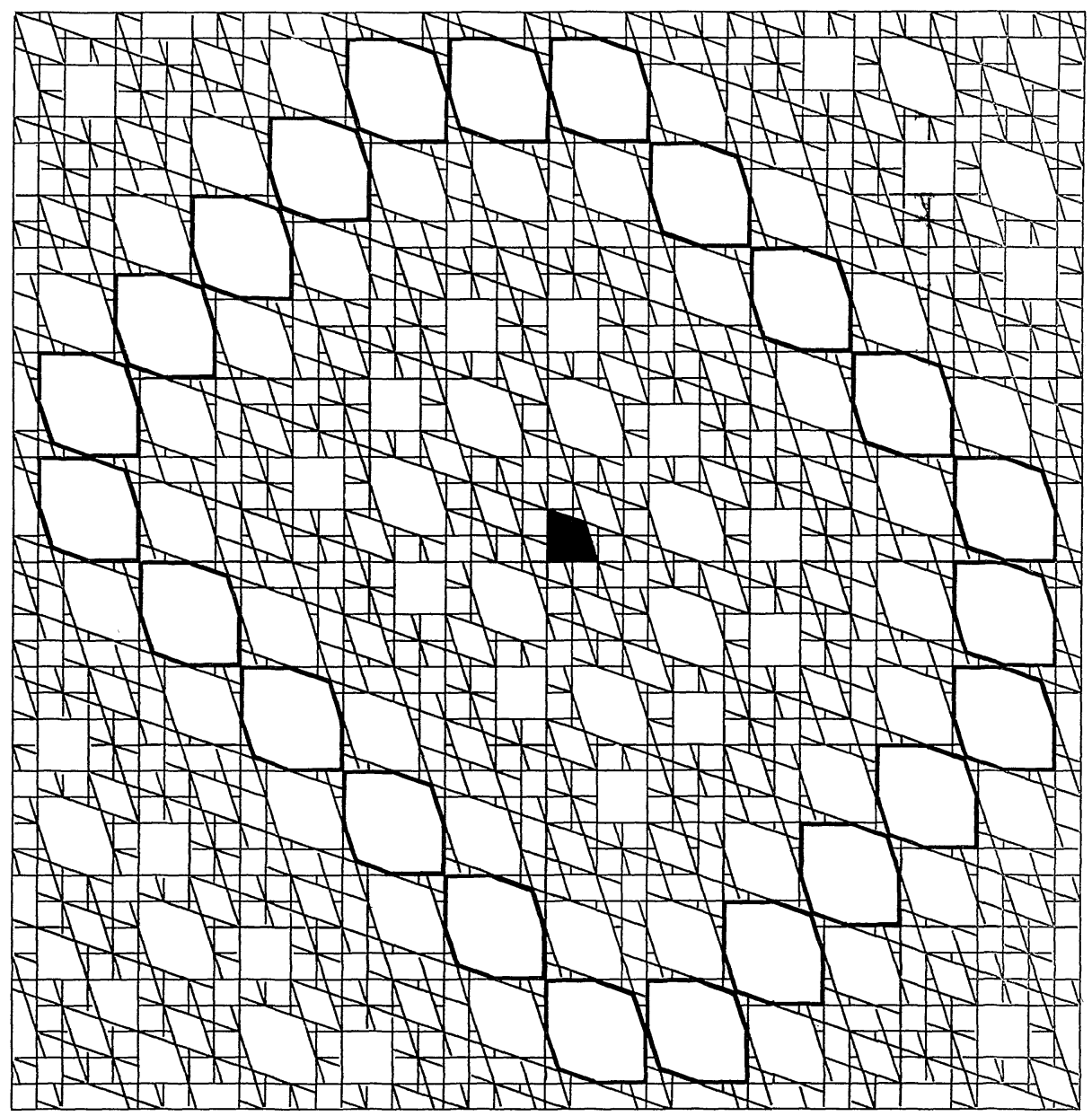

Fig. 5. The set $T$ in the case of a rational quadrilateral, with an invariant annulus of the first kind

number of sides. These annuli are the generalization of those seen in the case of the square and the triangle, and they guarantee the global stability of the mapping (see Fig. 5).

We begin analyzing the asymptotic structure of the orbits (excluding the special case in which some sides of $\Gamma$ are parallel). In what follows, the index $k$ is assumed to be periodic with period $2 N$ (with $W_{k+N} \equiv W_{k}$ and $\Gamma_{k+N} \equiv \Gamma_{k}$ ). At sufficiently large distance from $\Gamma$, the $N$ lines tangent to the sides of $\Gamma$ divide the plane into $2 N$ sectors $\Sigma_{k}$ (Fig. 2). The orientation of $\Gamma$ is chosen in such a way that $\phi^{2}$ rotates points clockwise near infinity, and the sectors $\Sigma_{k}$ are ordered accordingly. Let us denote the lines bounding $\Sigma_{k}$ by $W_{k-1}$ and $W_{k}$, respectively, and the sides of $\Gamma$ are labelled so that $\Gamma_{k} \subset W_{k}$.

Let $p \in \Sigma_{k}$, and define the vector $\xi_{k}$ by the equation

$$
2 \xi_{k}=\phi^{-1}(p)-\phi(p) .
$$


Note that $\xi_{k}$ depends on $k$ alone, and not on the choice of $p$ in $\Sigma_{k}$. Moreover $\xi_{k+N}=-\xi_{k}$.

We now construct the $2 N$-gon $\gamma$ with vertices $\xi_{k}$. This polygon is convex and centrally symmetric, by construction, and its sides are parallel to the lines $W_{k}$. The polygon $\gamma$, which is uniquely determined by $\Gamma$, will be called the fundamental polygon of $\Gamma$.

It is useful to consider the polygon $\gamma^{*}$ dual to $\gamma$, that is, the Legendre transformation of $\gamma$ [12]. We construct it by considering the family of lines $m y=l x+n$ which are tangent to $\gamma$. For any fixed value of $n$ (for which we do not make a specific choice here), we first consider the Legendre transformation of the $k^{\text {th }}$ axis of $\gamma$. Thus let $\xi_{k}$ have coordinates $\left(x_{k}, y_{k}\right)$. Then the $k^{\text {th }}$ axis of $\gamma$ is the segment joining $\left(x_{k}, y_{k}\right)$ and $\left(-x_{k},-y_{k}\right)$, which is transformed into the pair of lines $l=l(m)=m y_{k} / x_{k} \pm n / x_{k}$, which are parallel to $\xi_{k}$ (here we identify the new coordinates $l$ and $m$ with $y$ and $x$, respectively). These two lines bound a strip, which we call $H_{k} \cdot \gamma^{*}$ is now defined as the boundary of the intersection of the strips $H_{k}$, which is a convex and centrally symmetric polygon, whose sides are parallel to the axis of $\gamma$. Since the Legendre transformation is involutive (it transforms $\gamma^{*}$ into $\gamma$ ), the axis of $\gamma^{*}$ are parallel to the sides of $\gamma$. Moreover, if $d_{k}$ is the half-width of the strip $H_{k}$, then $\left|\xi_{k}\right| d_{k}=n$, independent of $k$ [this property will be used later, see (4.5)].

Let $\bar{W}_{k}$ be the line $\left(\neq W_{k}\right)$ parallel to $W_{k}$ and tangent to $\Gamma$. Also let $h_{k}=\Gamma \cap \bar{W}_{k}$. By analogy with $\Sigma_{k}$, we now denote by $\bar{\Sigma}_{k}$ the sector bounded by $\bar{W}_{k}$ and $\bar{W}_{k-1}$. Let $c_{1}$ be a point on $\bar{W}_{1}$ (at sufficiently large distance from $\Gamma$ ). From $c_{1}$ we draw a segment parallel to $\xi_{2}$ which meets the line $\bar{W}_{2}$ at a point $c_{2}$. By repeating this process $2 N$ times, we obtain a polygonal $\operatorname{arc} \delta$, with vertices $c_{1}, \ldots, c_{2 N}$. Let $L_{k}$ be the side of $\delta$ joining $c_{k-1}$ and $c_{k}$. Then $L_{k}$ lies in $\bar{\Sigma}_{k}$, and is parallel to $\xi_{k}$ as well as to $L_{k+N}$. We intend to show that the polygonal arc $\delta$ is in fact a polygon (its convexity is obvious from the way the $\xi_{k}$ 's are ordered). We may view the polygonal $\operatorname{arc} \delta$ as obtained from a centrally symmetric polygon similar to $\gamma^{*}$ via a sequence of parallel displacements of some of its axes, and we will now show that such shifts cannot transform a polygon into a polygonal arc.

Let $w_{k}$ be the line parallel to $\bar{W}_{k}$ through the point $\bar{W}_{1} \cap \bar{W}_{2}$ (Fig. 8). Then $w_{1}$ $=\bar{W}_{1}$ and $w_{2}=\bar{W}_{2}$. Let $\delta^{m}$ be the polygonal arc constructed in the same way as $\delta$, but using $\bar{W}_{1}, \ldots, \bar{W}_{m}, w_{m+1}, \ldots, w_{N}$ in place of $\bar{W}_{1}, \ldots, \bar{W}_{N}$. Let $c_{k}^{m}$ be its vertices. Then $\delta^{2}$ is similar to $\gamma^{*}$, that is, $\delta^{2}$ is a polygon, and $\delta^{N}=\delta$. We now proceed by induction on $m$, assuming that $\delta^{m-1}$ is a polygon and that $w_{m} \neq \bar{W}_{m}$. We denote the points $\delta^{m-1} \cap \bar{W}_{m}$ by $s_{m}$ and $s_{m+N}\left(s_{m}\right.$ is the nearest of the two to $\left.c_{m}^{m}\right)$, and the segments $c_{m}^{m} s_{m}$, $c_{m+i}^{m} c_{m+i}^{m-1}, c_{m+N}^{m} s_{m+N}$, by $g_{m}, g_{m+i}$, and $g_{m+N}$, respectively $(i=1, \ldots, N-1)$. Then none of these segments is degenerate. Moreover $g_{m}=g_{m+N}$. To see this, let the vector $\xi_{k}^{*}$ denote the $k^{\text {th }}$ vertex of $\gamma^{*}$, which is the dual of the side of $\gamma$ joining $\xi_{k+1}$ and $\xi_{k}$. Then $\xi_{k}^{*}$ is parallel to $W_{k}$, whence to $\bar{W}_{k}$ and to $w_{k}$. Since $g_{m+i}|| \xi_{m+i}^{*} \mid$ $=g_{m+i+1} /\left|\xi_{m+i+1}^{*}\right|, i=0, \ldots, N-1$ (by construction), and $\left|\xi_{m}^{*}\right|=\left|\xi_{m+N}^{*}\right|, \gamma^{*}$ being centrally symmetric, it follows that $g_{m}=g_{m+N}$. This implies that $\delta^{m}$ is a polygon.

We now show that $h_{k}$ is the midpoint of the segment $c_{k} c_{k+N}$. Suppose $c_{k} h_{k}$ $>h_{k} c_{k+N}$. Then also $c_{k+1} h_{k+1}>h_{k+1} c_{k+N+1}$, as easily verified. But then $c_{k+N} h_{k+N}$ $>h_{k+N} c_{k}$, and since $h_{k}=h_{k+N}$, we obtain a contradiction.

The lengths of $L_{k}$ and $L_{k+N}$ are related by the equality

$$
L_{k}=L_{k+N}+2 \Delta L_{k}\left|\xi_{k}\right|
$$

where $\Delta L_{k}$ is equal to 1 if $\Sigma_{k} \subset \bar{\Sigma}_{k}$, to -1 if $\Sigma_{k} \supset \bar{\Sigma}_{k}$, and to zero otherwise. 
The polygon $\delta$ is not invariant under $\phi$. The images of most of its points will either oscillate about it or spiral away from it. We shall therefore speak of annular and spiral orbits, respectively. More precisely, if $p \in L_{k}$, after changing sector its images lie on a line parallel to $L_{k+1}$ and whose distance from it is smaller or equal to $\Gamma_{k}$. Thus the maximal radial excursion of a point upon completing one revolution about $\Gamma$ cannot exceed twice the length of the perimeter of $\Gamma$. Asymptotically, the relative distance between $W_{k}$ and $\bar{W}_{k}$ goes to zero, and so does the relative size of $\Gamma$. Hence the polygon $\delta$ becomes similar to the dual fundamental polygon $\gamma^{*}$, for their respective sides and axes are pairwise parallel. In particular, all orbits at a sufficient distance from $\Gamma$ follow the shape of the dual fundamental polygon for many revolutions.

We shall now show that under suitable conditions the polygon $\delta$ can become the skeleton of an invariant annulus (see e.g. Fig. 4). We begin by seeking conditions on $\Gamma$ under which there will exist a denumerable sequence of polygons $\delta_{i}$ whose sides $L_{k, i}$, for fixed $i$, are all integral multiples of $\left|\xi_{k}\right|$. When this is the case, we shall speak of an integral polygon $\delta_{i}$ with integral sides $L_{k, i}$.

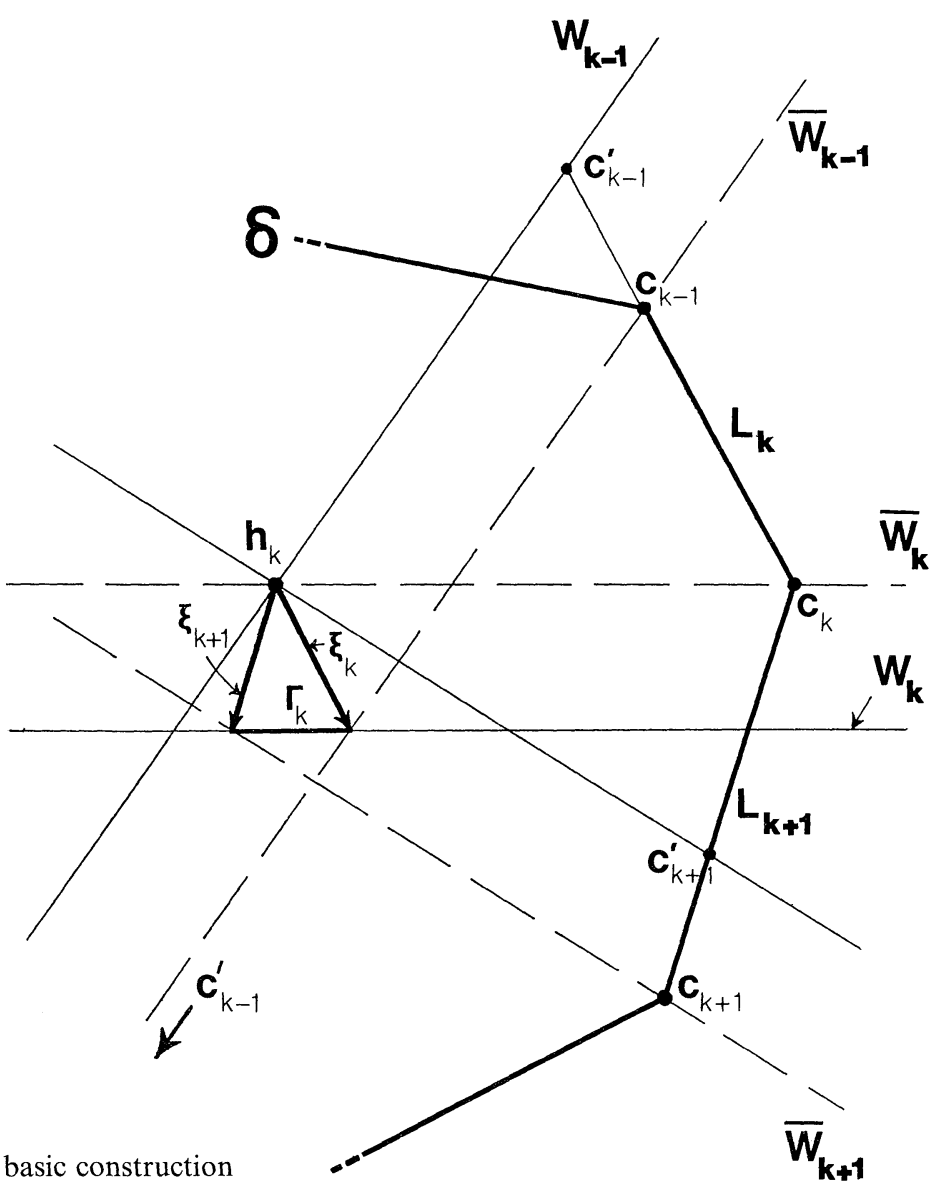

Fig. 6. The basic construction 
This requirement yields conditions on $\Gamma$, whence on $\gamma^{*}$. Let $\xi_{k}^{*}$ have coordinates $\left(x_{k}^{*}, y_{k}^{*}\right)$ and consider the $N$ determinants,

$$
m_{k}=\left|\operatorname{Det}\left(\xi_{k}^{*}, \xi_{k-1}^{*}\right)\right|=\left|x_{k}^{*} y_{k-1}^{*}-y_{k}^{*} x_{k-1}^{*}\right|, \quad k=1, \ldots, N,
$$

i.e. $m_{k}$ is twice the area of the $k^{\text {th }}$ sectors of $\gamma^{*}$. We say that $\Gamma$ is weakly rational, if the linear space generated by the $m_{k}$ 's over $\mathbb{Q}$ is isomorphic to $\mathbb{Q}$. When this is the case, we can always take the $m_{k}$ 's to be coprime integers.

Let now $\Gamma$ be weakly rational, and consider again the basic construction (Fig. 6). Prolong (if necessary) the segments $L_{k}$ and $L_{k+1}$, to meet $W_{k-1}$ and $W_{k+1}$ in the points $c_{k-1}^{\prime}$ and $c_{k+1}^{\prime}$, respectively. Since the triangles $h_{k} c_{k} c_{k-1}^{\prime}$ and $h_{k} c_{k} c_{k+1}^{\prime}$ are now equivalent to the $k^{\text {th }}$ and $k+1^{\text {th }}$ sectors of $\gamma^{*}$, respectively, we can choose $c_{k}$ on $\bar{W}_{k}$ in such a way that their areas be $m_{k} / 2$ and $m_{k+1} / 2$, respectively. We define

$$
Q_{k}=L_{k} /\left|\xi_{k}\right|, \quad P_{k}=Q_{k}-\Delta L_{k}
$$

Then $\delta$ is integral precisely when the $P_{k}$ 's are integers. Since $\left|\xi_{k}\right| P_{k}$ is the length of the segment $c_{k} c_{k-1}^{\prime}$, and $\left|\xi_{k+1}\right| P_{k+1}$ that of $c_{k} c_{k+1}^{\prime}$, we obtain the relations

$$
\left|\xi_{k}\right| P_{k} d_{k}=m_{k}, \quad\left|\xi_{k}\right| d_{k}=\left|\xi_{k+1}\right| d_{k+1},
$$

$d_{k}\left(d_{k+1}\right)$ being the distance of $L_{k}\left(L_{k+1}\right)$ from $h_{k}$. Since the triangles $h_{k} c_{k} c_{k+1}^{\prime}$ and $h_{k+1} c_{k+1} c_{k}^{\prime}$ are congruent, Eqs. (4.5) are valid for all $k$. From (4.5) one obtains $m_{k+1} P_{k}=m_{k} P_{k+1}$, whose smallest solution is just $P_{k}=m_{k}$, since the $m_{k}$ 's do not have common divisors. Then we obtain at once an infinity of integral polygons $\delta_{i}$, whose sides are given by

$$
Q_{k, i}=i m_{k}+\Delta L_{k}, \quad i=1,2, \ldots .
$$

Before constructing invariant annuli about the integral polygons $\delta_{i}$, we shall characterize more precisely those regions (near infinity) where $\phi^{2}(p)=p+2 \xi_{k}$. Let $W_{k}^{\prime}$ be the line obtained by reflecting $W_{k}$ with respect to $\bar{W}_{k}$. The lines $W_{k}$ and $W_{k}^{\prime}$ bound a strip which we call $R_{k}$. Then the sector $\Sigma_{k}^{\prime}=\left(\Sigma_{k} \cup R_{k-1}\right)-R_{k}$ has the desired property. The sectors $\Sigma_{k}, \bar{\Sigma}_{k}$, and $\Sigma_{k}^{\prime}$ are related in different ways. There are four possible cases, as detailed below:

\begin{tabular}{lllllll}
\hline Type & $\Delta L_{k}$ & $\Sigma_{k} \supset \bar{\Sigma}_{k}$ & $\Sigma_{k} \subset \bar{\Sigma}_{k}$ & $\Sigma_{k} \supset \Sigma_{k}^{\prime}$ & $\Sigma_{k} \subset \Sigma_{k}^{\prime}$ & $h_{k}=h_{k-1}$ \\
\hline I & -1 & yes & no & yes & no & no \\
II & +1 & no & yes & no & yes & no \\
III & 0 & no & no & no & no & yes \\
IV & 0 & no & no & yes & yes & yes \\
\hline
\end{tabular}

If the $k^{\text {th }}$ sector is of Type I (Type III), the $(k+N)^{\text {th }}$ sector is of Type II (Type IV), and vice-versa. Since $h_{k}=h_{k-1}$ for Type III and IV, Type I (whence Type II) sectors must always occur.

Proposition 1. All orbits of a weakly rational polygon are bounded.

Let a polygon $\delta_{i}$ be integral. We first consider the case in which all $Q_{k, i}$ are even (whence $i$ must be odd). We now show that an invariant annulus can be constructed as the union of all images of a fundamental polygon $\gamma$ whose center 


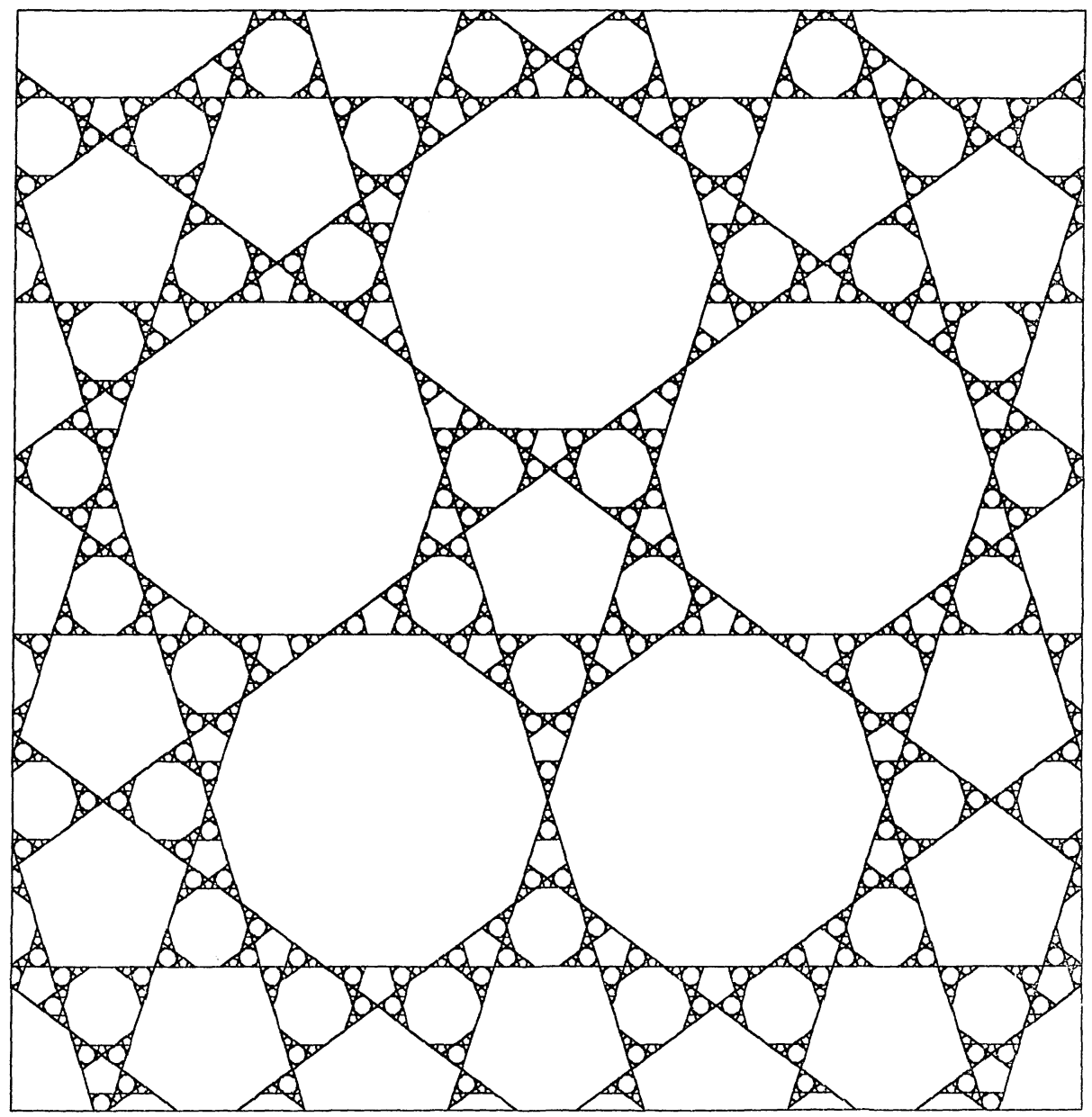

Fig. 7. The set $T$ for the pentagon

coincides with a vertex of $\delta_{i}$, say $c_{k-1}$. Then $\gamma$ is tangent to $W_{k-1}$ as well as to $W_{k-1}^{\prime}$, that is, $\gamma$ is contained in the strip $R_{k-1}$, whence in $\Sigma_{k}^{\prime}$. Within each sector $\Sigma_{k}^{\prime}$, the map $\phi^{2}$ displaces $\gamma$ along the direction of the $k^{\text {th }}$ axis of $\gamma$, by definition. Since this axis is twice as long as $\xi_{k}, \phi^{2}(\gamma)$ touches $\gamma$ at the corresponding vertex, and moreover $\gamma$ and $\phi^{2}(\gamma)$ do not have any other point in common. Since $Q_{k, i}$ is even, by assumption, the polygon $\phi^{Q_{k, i}}(\gamma)$ is centered on the vertex $c_{k}$ of $\delta_{i}$, and therefore it is also tangent to $W_{k}$ as well as to $W_{k}^{\prime}$, i.e. lies within the strip $R_{k}$. By repeating this procedure for all sectors, the chain of fundamental polygons with centers belonging to $\delta_{i}$ closes up, and we obtain an "annulus" consisting of $M_{i}$ fundamental polygons, where

$$
M_{i}=\sum_{k=1}^{N}\left(Q_{k, i}-\Delta L_{k}\right)=i \sum_{k=1}^{N} m_{k} .
$$




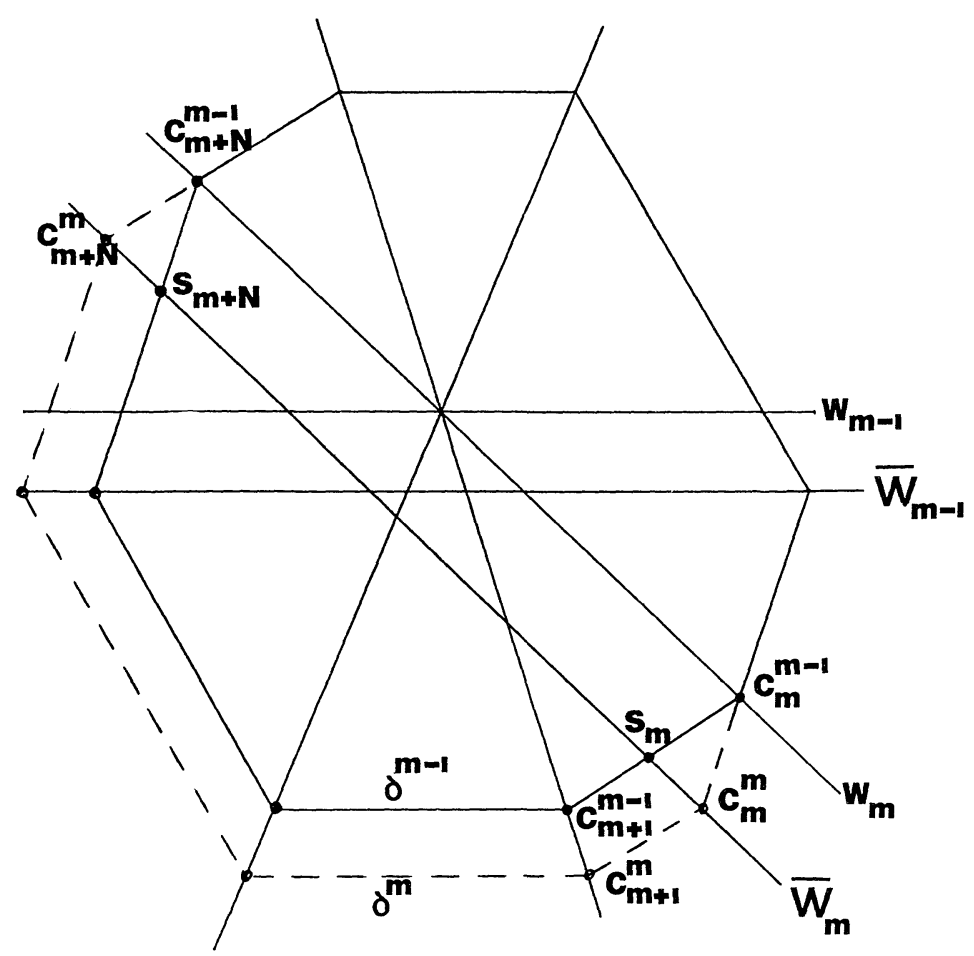

Fig. 8. Construction of the polygon $\delta$ from the dual fundamental polygon $\gamma^{*}$

Thus each polygon is invariant under $\phi^{M_{i}}$, and since none of them intersect $W$, they all belong to $S$ from (3.1). Annuli constituted by fundamental polygons will be referred to as annuli of the first kind.

The case in which at least one of the $Q_{k, i}$ is odd is treated in an analogous way, but now the annuli are constructed by suitably assembling polygons identical to $\Gamma$ itself (annuli of the second kind). More precisely, to construct the annulus, we begin by replacing the fundamental polygon centered on $c_{k-1}$ with two polygons identical to $\Gamma$ (Fig. 9). One of these (which we still call $\Gamma$ ) is obtained by translating $\Gamma$ in such a way that $h_{k-1}$ coincides with $c_{k-1}$, while the other (which we denote by $\left.\Gamma^{\prime}\right)$ is the reflection of the former with respect to $c_{k-1}$. Then $\Gamma \cup \Gamma^{\prime}$ is tangent to both $W_{k}$ and $W_{k}^{\prime}$, i.e. it belongs to $R_{k-1}$. Again $\Gamma \cup \Gamma^{\prime}$ touches its image under $\phi^{2}$ at precisely one point. We now assume that $i$ is even in (4.6), that is, $Q_{k, i}$ has the same parity as $\Delta L_{k}$. For Type III and IV sectors, $\Delta L_{k}=0$, whence $Q_{k, i}$ is even, and the $Q_{k, i}{ }^{\text {th }}$ iterate of $\Gamma \cup \Gamma^{\prime}$ lies within $R_{k}$, being tangent to both $W_{k}$ and $W_{k}^{\prime}$, by construction. Moreover in this case $h_{k-1}=h_{k}$, so that this section of the annulus is properly joined to the successive one. For Type I and II, $Q_{k, i}$ is odd, so that the image of $\Gamma \cup \Gamma^{\prime}$ in the strip $R_{k}$ is congruent to $\Gamma^{\prime} \cup \phi^{2}(\Gamma)$ (for Type I), or to $\Gamma \cup \phi^{2}\left(\Gamma^{\prime}\right)$ (for Type II), which is tangent to $W_{k}$ and $W_{k}^{\prime}$. Since the image of $\Gamma^{\prime}$ in $R_{k}$ is now the reflection of that of $\Gamma$ with respect to $h_{k}$, we can again proceed further, and complete the annulus.

The number $M_{i}$ of polygons in an annulus of the second kind is given by

$$
M_{i}=2 \sum_{k=1}^{N}\left(Q_{k, i}-\Delta L_{k}\right)=2 i \sum_{k=1}^{N} m_{k} .
$$




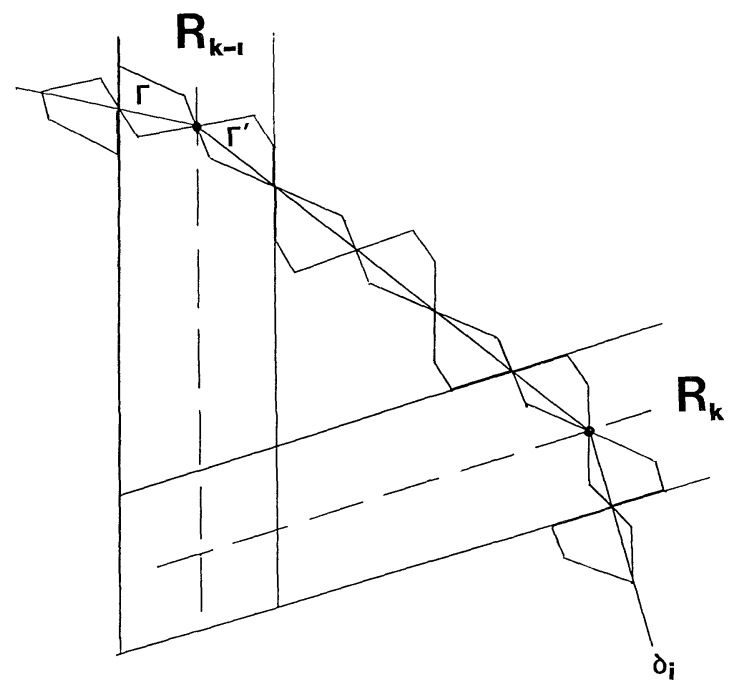

Fig. 9. Joining segments of an invariant annulus of the second kind at the vertices of an integral polygon $\delta_{i}$. This construction refers to the case of Type I sectors

Now we note that both inner and outer boundaries of an annulus are invariant under the mapping. This property is obviously true for those elements of the boundary which belong to the interior of each sector, and it holds also on the discontinuity lines, from the way the map was defined on them (see Sect. 3). For this reason all orbits within an annulus are bounded. In conclusion, we have invariant annuli of the second kind for even $i$ [in (4.6)], and annuli of the first kind, or no annuli at all, for odd $i$. Our assertion now follows from the fact that invariant annuli can be found at any arbitrarily large distance from $\Gamma$. Q.E.D.

From the above considerations we can also conclude that the fundamental polygon itself is the largest member of the family $\left\{S_{i}\right\}$. This is because no polygon containing $\gamma$ can have congruent images within each strip $R_{k}$, and therefore it will be "cut" by the discontinuity lines. Thus the following inequality holds

$$
\mu\left(S_{i}\right) \leqq \mu(\delta),
$$

which is independent on $\Gamma$ being weakly rational.

\section{Finite Structures}

In Fig. 5 we display the set $T$ for the case of a rational quadrilateral, with parameters $m_{k}=4,2,3,2$, respectively. Here the stability is ensured by annuli of both the first and the second kind, the innermost one being visible near the edge of the picture. In addition the measure of the $S_{i}$ 's is bounded away from zero, and only a finite number of different shapes is available. In other words, all orbits are periodic (see below) and have a maximal period in any bounded domain of the plane. The strips between annuli model the regions of instability of smooth 
systems. Irregular orbits are represented by spiral orbits, which perform bounded radial excursions and correspond to small polygons. Within each strip we also find regions of stability, represented by annular disconnected sets of polygons. On the whole, we note that $T$ accumulates more in some regions than others.

In the case of the regular pentagon (Fig. 7) there are annuli of both the first and the second kind. The map is still fully periodic, but now the period can be arbitrarily large, that is the $S_{i}$ 's arbitrarily small. The structure of $T$ is hierarchical, and is invariant under an obvious renormalization. The map generated by the pentagon offers the simplest example of a nested set of stable and unstable periodic orbits which characterize smooth nonintegrable maps.

The case of the quadrilateral discussed above constitutes one example of a finite approximation to some smooth nonintegrable map. It is therefore desirable to find conditions under which similar properties are shared by rational $\mathrm{N}$-gons with arbitrarily large $N$. It is clearly necessary and sufficient to require that the measure of the $S_{i}$ 's be bounded away from zero. To this end we note that, despite its complexity, $T$ can be embedded in a set with simple geometrical properties. Indeed all iterates of a given line $W_{k}$ are parallel to it, so that $T$ itself is embedded in $N$ families of parallel lines. Construction of these lines involves reflection of each $W_{k}$ with respect of all vertices $v_{i}$ of $\Gamma$. More precisely $\phi\left(W_{k}\right) \cup \phi^{-1}\left(W_{k}\right)$ belongs to the union of lines parallel to $W_{k}$ at a distance $2 d_{k, i}$, where $d_{k, i}$ denotes the altitude of the vertex $v_{i}$ with respect to $W_{k}$. This set of lines will then be reflected again from all vertices and so forth, ad infinitum. The distance between neighboring parallel lines of the resulting set is bounded away from zero if and only if the linear space generated by the vectors $\xi_{k}$ over $\mathbb{Q}$ is isomorphic to $\mathbb{Q}^{2}$ (for then the $\xi_{k}$ 's can be chosen in such a way as to have rational coordinates). When this is the case we say that the polygon $\Gamma$ is rational. Rational polygons are dense among all convex curves (with the standard topology).

We note that rational implies weakly rational. To prove this, it is sufficient to show that when $\Gamma$ is rational, the linear spaces (over $\mathbb{Q}$ ) generated by the $\xi_{k}$ 's and $\xi_{k}^{*}$ 's, respectively, are isomorphic. Using the Legendre transformation, we find that $\xi_{k}^{*}=n\left(\xi_{k+1}-\xi_{k}\right) / \operatorname{Det}\left(\xi_{k+1}, \xi_{k}\right)$. This linear transformation is the required isomorphism, since it is invertible and its coefficients generate a linear space isomorphic to Q. Weak rationality implies rationality only for $N \leqq 4$. The case $N=3$ is trivial. As to $N=4$, we observe that the fundamental polygon of any quadrilateral can be put in "canonical" form: $\xi_{1}=(-1,0), \xi_{2}=(-1,1), \xi_{3}=(0,1)$. Then, using the isomorphism described above, it is a simple matter to verify that the weak rationality of $\Gamma$ implies that $\xi_{4}$ has rational coordinates, i.e. $\Gamma$ is rational.

Examples of polygons which are weakly rational but not rational are provided by the regular $N$-gons with $N \neq 2,3,4,6$. Indeed, the linear space generated by the corresponding vectors $\xi_{k}$ is isomorphic to the additive group of the $N^{\text {th }}$ cyclotomic field (the extension of $\mathbb{Q}$ generated by the $N^{\text {th }}$ roots of unity), whose dimension over $\mathbb{Q}$ is $\Phi(N), \Phi$ being the Euler $\Phi$-function [13]. Now $\Phi(N)$ is smaller or equal to 2 only for $N=2,3,4$, and 6 , which are precisely the solvable cases described in Sect. 3. At this stage the above claim is justified only for odd $N$ (here $\gamma$ is a $2 N$ gon, but the $N^{\text {th }}$ and $2 N^{\text {th }}$ cyclotomic fields are the same, when $N$ is odd). Regular polygons with an even number of sides have opposite sides parallel, and have been excluded from our analysis. We remark, however, that the procedure we have 
described to construct invariant annuli can be suitably generalized to this case (also note that for a regular $2 N$-gon, $\Gamma=\gamma$ ).

The results of the previous section are strengthened by the following:

Proposition 2. All orbits of a rational polygon are periodic, and most of them Lyapounov stable. In addition their period is bounded from above in any bounded region of the plane.

For orbits in $S$, the validity of this statement can be deduced from the Lemma and Proposition 1. If $p \in T$, the orbit through $p$ is obtained by reflecting $p$ with respect to the vertices of $\Gamma$, as well as to the midpoints of the sides of $\Gamma$. Since $\Gamma$ is rational, all these points belong to a two-dimensional lattice, and so do all images of $p$. Now all orbits are bounded (including those in $T$ ), and therefore the set of all images of $p$ is finite, i.e., any orbit in $T$ is periodic. From the fact that $\mu\left(S_{i}\right)$ is bounded away from zero one can also establish that the family $\left\{S_{i}\right\}$ inside an annulus can contain only a finite number of elements.

It is worth noting that the boundness and periodicity of the orbits in $T$ depend crucially on the way the map was defined on the discontinuity lines.

\section{Concluding Remarks}

The mapping of a rational polygon enjoys the remarkable property of supporting only periodic orbits. The importance of this class of orbits for the analysis of irregular motions has been long recognized and emphasized, most notably by Poincaré, who wrote [14]

“...These periodic solutions are so valuable for us because they are, so to say, the only breach by which we may attempt to enter an area heretofore deemed inaccessible."

In our case, the algorithmic content of the map of a rational polygon is finite, so that the problem of determining the evolution of all orbits for all times lies within computational range. We could therefore speak of "algorithmic integrability" [8, 15]. The situation here is somewhat similar to that of maps acting on bounded domains of integers, all whose orbits are also periodic (a fine example of one such map will be found in [16]). This analogy becomes more stringent if one considers that integral maps can still be viewed as acting on the continuum, by permuting squares rather than individual points. In fact with this device all orbits become Lyapounov stable, with the sole exception of those belonging to the discontinuity set, which is now an orthogonal lattice. However, unlike the case of integral maps, the discontinuity set of polygonal billiards is system-dependent, and moreover it is shaped by the unstable manifold of some nearby nonintegrable system.

In closing we would like to remark that the limit $N \rightarrow \infty$ should be taken with much care. As a symptom of singular behaviour we note that, when the number of sides of $\Gamma$ increases, the invariant annuli we have constructed in Sect. 4 are forced to migrate to infinity. Our results, however, do not rule out the possibility that other kinds of invariant sets could provide narrower bounds for the radial excursion of the orbits. 
Acknowledgements. This problem was suggested to us by J. K. Moser. We also feel indebted to B. H. Newmann (the inventor of dual billiards) for a stimulating and enjoyable discussion, and to

O. E. Lanford III for very constructive comments on the manuscript.

\section{References}

1. Moser, J.K.: Stable and random motions in dynamical systems. Princeton, NJ: Princeton University Press 1973

2. Siegel, C.L., Moser, J.K.: Lectures on celestial mechanics. Berlin, Heidelberg, New York: Springer 1971, p. 183

3. Moser, J.K.: Lectures on Hamiltonian systems. Mem. Am. Math. Soc. 81, 1 (1968)

4. Henon, M., Wisdom, J.: The Benettin-Strelcyn oval billiard revisited. Physica 8 D, 157 (1983)

5. Devaney, R.L.: A piecewise linear model for the zones of instability of an area-preserving map. Physica 10 D, 387 (1984)

6. Zemlyakov, A.N., Katok, B.: Mat. Zametki 18, 291 (1975) [English transl.: Math. Notes 18, 760 (1976)]

7. Richens, P., Berry, M.: Pseudointegrable systems in classical and quantum mechanics. Physica 2 D, 495 (1981)

8. Eckardt, B., Ford, J., Vivaldi, F.: Analytically solvable dynamical systems which are not integrable. Physica 13 D, 339 (1984)

9. Hammer, P.C.: Unsolved problems. Proc. Symp. Pure Math., Vol. VII. Am. Math. Soc. (1963)

10. Moser, J.K.: Is the solar system stable? Math. Intell. 1, 2, 65 (1976)

11. Greene, J.M.: Two-dimensional measure-preserving maps. J. Math. Phys. 9, 760 (1968)

12. Arnold, V.I.: Mathematical methods of classical mechanics. Moskow: Nauka 1974 [English transl.: Berlin, Heidelberg, New York: Springer 1968, p. 61]

13. Marcus, D.A.: Number fields, p. 18. Berlin, Heidelberg, New York: Springer 1977

14. Poincaré, H.: Les méthodes nouvelles de la mécanique céleste. Paris: Gauthier-Villars 1892. NASA Translation TT-F450, Washington 1976. Art. 36

15. Ford, J.: How random is a coin toss? Physics Today 36 (4), 40 (1983)

16. Rannou, F.: Numerical study of discrete plane area-preserving mappings. Astron. Astrophys. 31, 289 (1974)

Communicated by O. E. Lanford

Received December 27, 1984; in revised form September 9, 1986 